\title{
Subjective duration: A functional measurement analysis
}

\author{
DONALD A. BLANKENSHIP \\ Goethe Universität, 6000 Frankfurt am Main, West Germany \\ and \\ NORMAN H. ANDERSON \\ University of California, San Diego, La Jolla, California 92093
}

\begin{abstract}
Subjects judged the total duration of two intervals of time that varied from 0 to 30 sec. The data obeyed the parallelism prediction of a simple weighted adding model. According to functional measurement methodology, this observed parallelism provides joint support for the adding model and for the assumption that the graphic rating response provides a valid interval scale. Furthermore, parallelism allowed construction of the psychophysical time function. The obtained function was essentially linear in the range 0-30 sec. This result is compared with results of previous studies, with particular regard to the method of fractionation. The usefulness of establishing a validated interval response measure for further studies of time perception was pointed out.
\end{abstract}

Much research has been directed toward the discovery of factors affecting subjective impressions of duration, and particularly toward the determination of the psychophysical time function (see reviews by Doob, 1971; Fraisse, 1963; Ornstein, 1970). Two experimental methods have predominated in this research: the method of reproduction, in which the subject is asked to reproduce the duration of some previous event; and the method of comparison, in which the subject is asked to express the duration of the event as a fraction or multiple of a "standard" event or of some objective unit of duration, such as a second.

Both methods suffer serious weaknesses. The method of reproduction is untrustworthy because it necessarily confounds factors affecting the subjective durations of the initial, presentation event and the subsequent, reproduction event; differences in attentional and set characteristics of these two events cannot be controlled and may introduce time-order errors. The method of comparison is suspect, because the assumption that the chosen response measure is an interval scale of subjective duration has lacked empirical justification.

A possible solution to these problems is given by the method of functional measurement (Anderson, 1974a). Suppose that a subject experiences two events, and then reports his impression of the total

This work was supported by National Science Foundation Grant BMS 74-19124. We wish to thank Diane Cuneo for her assistance in this work. Requests for reprints should be sent to D. A. Blankenship, Institut für Psychologie, Goethe Universität, 6000 Frankfurt am Main, West Germany. duration of the two events. It is assumed that his response follows a simple weighted sum model:

$$
R_{i j}=A\left(w_{1} d_{i}+w_{2} d_{j}\right)+B,
$$

Where $\mathbf{R}$ is the subject's response (in arbitrary numerical units), $d_{i}$ and $d_{j}$ are his subjective impressions of the durations of the two events, and $w_{1}$ and $w_{2}$ are weights that reflect the subjective importance of the first and second events upon the total judgment. The parameters $A$ and $B$ are included to allow for linear transformation of the subjective judgment to the particular response scale being used.

This model has a very simple analysis. Let the event pairs be constructed from a row by column design, in which the row factor corresponds to the first event and the column factor corresponds to the second event. If the model is correct, and if the response measure is an equal-interval scale, then the data will exhibit a pattern of parallelism. That is, if the data are plotted in the usual row by column form, as in Figure 1 below, then they will form a set of parallel curves.

Observed parallelism thus accomplishes three ends (Anderson, 1974a, p. 221):

(1) It supports the validity of the integration model.

(2) It supports the assumption that the observed response is on an interval scale.

(3) It allows construction of interval scales of the stimulus directly from the marginal means of the factorial design.

The parallelism prediction could fail for either of two reasons. First, the observed response might not be an interval scale. Indeed, the graphic rating 


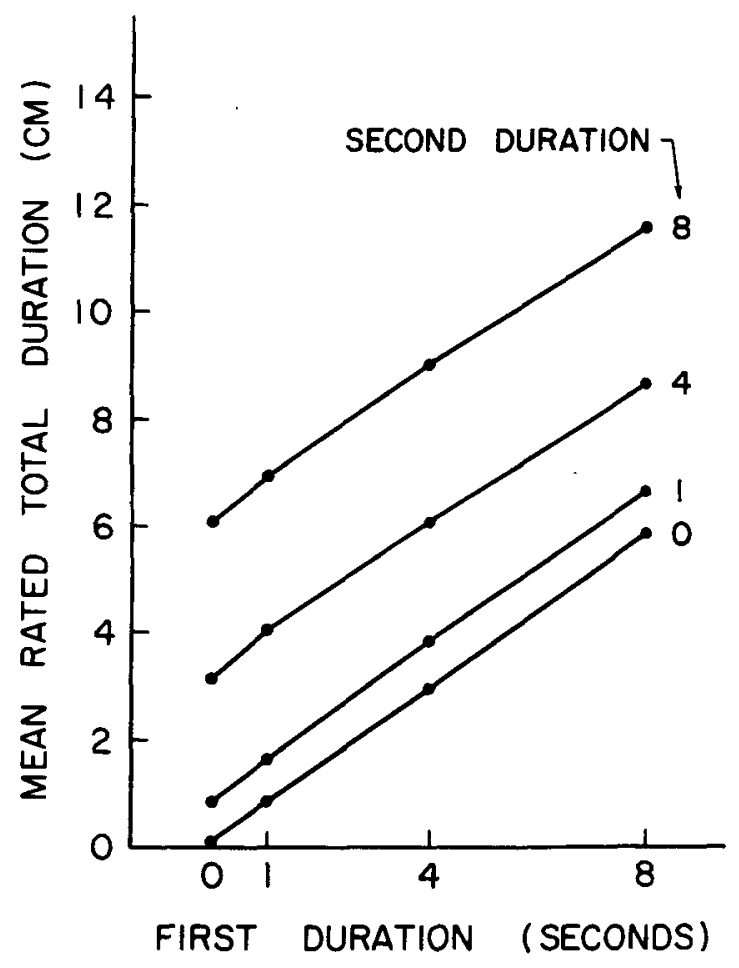

Figure 1. Mean rated total duration of time intervals in Part 1.

method employed here has been claimed by Stevens (e.g., 1974) to constitute a partition-type scale that is severely biased and invalid for prothetic dimensions such as time. For this reason, success of the parallelism prediction would be important for direct scaling methods.

Second, the parallelism prediction could fail if the model was wrong. For example, the integration operation might be subadditive, following a law of diminishing returns. Or the integration task could well introduce contrast effects, with the second duration being perceived as shorter or longer according as the first duration was comparatively long or short. Or perhaps the integration task might even introduce a "partition" bias, as has been claimed by Stevens (1974).

For these reasons, success of the parallelism prediction has substantive interest. Furthermore, if the integration model can be established, then it becomes a useful tool for scaling in other studies of perceived duration, some of which are noted in the discussion.

\section{METHOD}

The subject's task was to judge the combined duration of two intervals of time. Subjects were encouraged to report their subjective impressions of the intervals, rather than attempt to make objectively accurate judgments.

\section{Experimental Design}

The experiment was conducted in two parts. In Part 1 , the first and second time intervals were $0,1,4$, or $8 \mathrm{sec}$. The interstimulus interval was 1,3 , or $5 \mathrm{sec}$. The complete design was thus a 4 by 3 by 4 factorial with 48 different stimulus combinations.
Each subject completed two replications of this design on each of 3 successive days.

In Part 2, a 3 by 10 design was used. The first time interval was 2,6 , or $10 \mathrm{sec}$; the second time interval was $0,1,2,4,8,12$, $16,20,25$, or $30 \mathrm{sec}$. The interstimulus interval was fixed at $2 \mathrm{sec}$. Each subject completed three replications of the design on 1 day, and two replications on the following day.

In both parts, subjects were instructed to regard the first replication as practice, and the data from these replications were not analyzed.

\section{Procedure}

In the experimental situation, the subject sat in a dimly lit room in front of a CRT screen. Each trial started when the word "begin" appeared on the screen. This was followed by a blank screen for $2 \mathrm{sec}$, a small stimulus pattern for a variable time (first time interval), a blank screen for a variable time (interstimulus interval), a stimulus pattern for a second variable time (second time interval), a blank screen for $1 \mathrm{sec}$, and finally a $15.5-\mathrm{cm}$ horizontal line that served as a response scale.

The response line was labeled "zero time" and "very long time" at its left and right ends, respectively. The subject responded by turning a knob that moved a short vertical arrow just above the response line. After making his response, the subject flicked a switch that terminated that trial and initiated the next. The entire sequence of trials was controlled by a PDP-12 computer, which also recorded the subject's responses.

Special end anchors were used in the instruction period to define the usage of the response scale. These were time intervals of 0 and $20 \mathrm{sec}$ for Part 1, and 0 and $45 \mathrm{sec}$ for Part 2. The end anchors were presented again before each successive replication.

The stimulus pattern used to define the first and second time intervals was a 5 by 5 square matrix of dots, about $2 \mathrm{~cm}$ on a side, except for the 0 -sec interval. Since even very short presentations of the dot pattern seemed to have appreciable subjective duration, the 0 -sec duration was defined by a plus sign of duration $.07 \mathrm{sec}$ which the subject was instructed to treat as zero duration.

Subjects were eight students who were paid for their services. One subject failed to return for Part 2.

\section{RESULTS}

\section{Model Analysis}

Figures 1 and 2 show the mean responses from the two parts of the experiment. The critical aspect

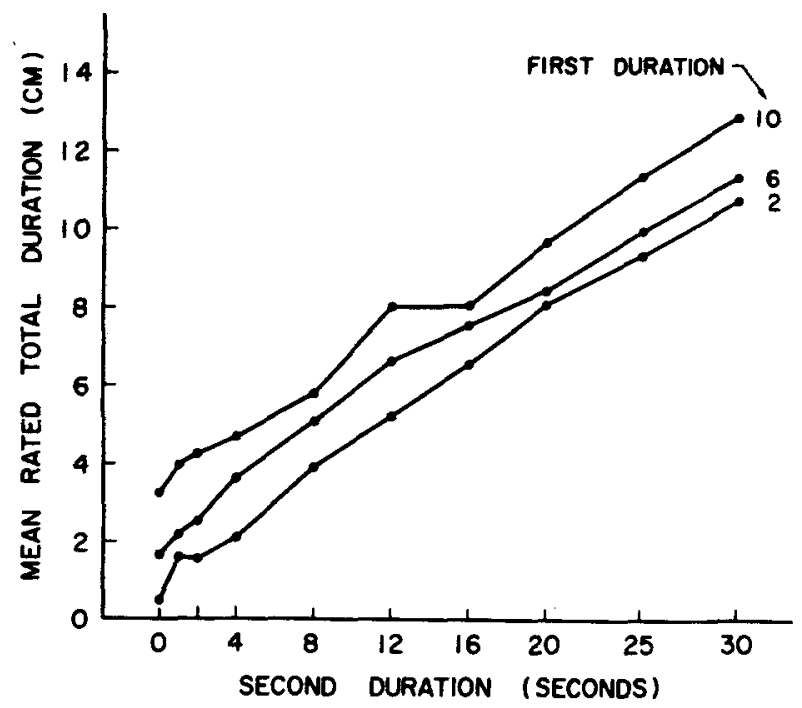

Figure 2. Mean rated total duration of time intervals in Part 2. 
Table 1

Summary of F Ratios and Error Mean Squares

\begin{tabular}{|c|c|c|c|c|c|c|c|c|}
\hline \multirow[b]{2}{*}{ Subject } & \multicolumn{4}{|c|}{ Part 1} & \multicolumn{4}{|c|}{ Part 2} \\
\hline & First Time & Second Time & Interaction & $\mathrm{MS}_{\mathrm{e}}$ & First Time & Second Time & Interaction & $\mathrm{MS}_{\mathrm{e}}$ \\
\hline BC & 210 & 246 & .34 & 2.06 & 64 & 181 & 1.47 & .94 \\
\hline SW & 559 & 522 & $5.97^{*}$ & 1.09 & 74 & 162 & .89 & 1.26 \\
\hline GO & 181 & 285 & $2.38^{*}$ & 1.93 & 11 & 75 & 1.30 & 1.64 \\
\hline DP & 331 & 421 & .96 & 1.11 & 30 & 69 & .54 & 2.72 \\
\hline RT & 218 & 270 & 1.80 & 2.03 & 52 & 121 & 1.10 & 1.47 \\
\hline KF & 300 & 331 & 1.01 & 1.32 & 8 & 33 & 1.24 & 1.97 \\
\hline DM & 75 & 74 & 1.05 & 2.04 & 31 & 76 & .63 & 1.51 \\
\hline JK & 164 & 149 & .58 & 2.47 & & & & \\
\hline Group & 217 & 203 & .57 & 2.83 & 51 & 128 & $2.04^{*}$ & 1.38 \\
\hline
\end{tabular}

$* p<.05$

of these data concerns the parallelism prediction of the adding model in Equation 1. By visual inspection, both figures appear to conform to the parallelism pattern, although the data for Part 2 are somewhat more variable, reflecting the greater difficulty that the subjects had in handling the much longer time intervals.

Parallelism is equivalent to zero interaction in the analysis of variance. The results of this statistical test of the model are summarized in Table 1. In the single-subject analyses, two subjects, G.O. and S.W., show significant interactions in Part 1 , but no subject shows significant interaction in Part 2. The two significant interactions reflected a moderate tendency for the curves either to converge (G.O.) or diverge (S.W.) at longer first intervals. Further work may need to consider individual differences in more detail.

In the group analyses in the last line of Table 1 , the interaction term for Part 1 is not significant, $F(9,63)=.57$, but the interaction term for Part 2 is significant, $F(18,108)=2.04$. The interpretation of the latter interaction is uncertain: it may reflect a real defect in the model, or only a mild nonlinear bias in the response scale. Inspection of Figure 2 shows no systematic trend in the nonparallelism, but rather a tendency for the larger deviations from parallelism to occur when one of the curves falls near the center of the response scale. That suggests a response bias interpretation, since the response pointer was always centered on the response line before a new trial began.

Although this response nonlinearity could be removed by application of the monotone scaling procedure of functional measurement (Anderson, $1974 a$, p. 277), it seems preferable to present the raw data without transformation, in order to facilitate any alternative interpretation. It may be noted, however, that the deviations from parallelism do not seem large enough to require any serious qualification of the conclusions; the interaction accounts for only $0.5 \%$ of the total systematic variance.

\section{Psychophysical Function}

If the model is accepted, then interval scales of subjective duration are immediately available. The least-squares estimates of the scale values are the marginal means of the factorial design (Anderson, 1974a, Section II.A). The function relating these subjective durations to objective duration is the psychophysical time function. Graphically, these psychophysical time functions correspond directly to the average curves in Figures 1 and 2, respectively.

These psychophysical time functions from Parts 1 and 2 are shown in Figure 3. The curve for Part 2 is nearly linear, with a slight downward bowing; deviations from linearity were not significant, either in the quadratic or in the pooled higher order components. The psychophysical function for Part 1 is almost exactly linear.

The apparent difference between the two psychophysical functions in Figure 3 is only apparent, a reflection of the fact that the subjective scale has different zero and unit in the two cases. The zero

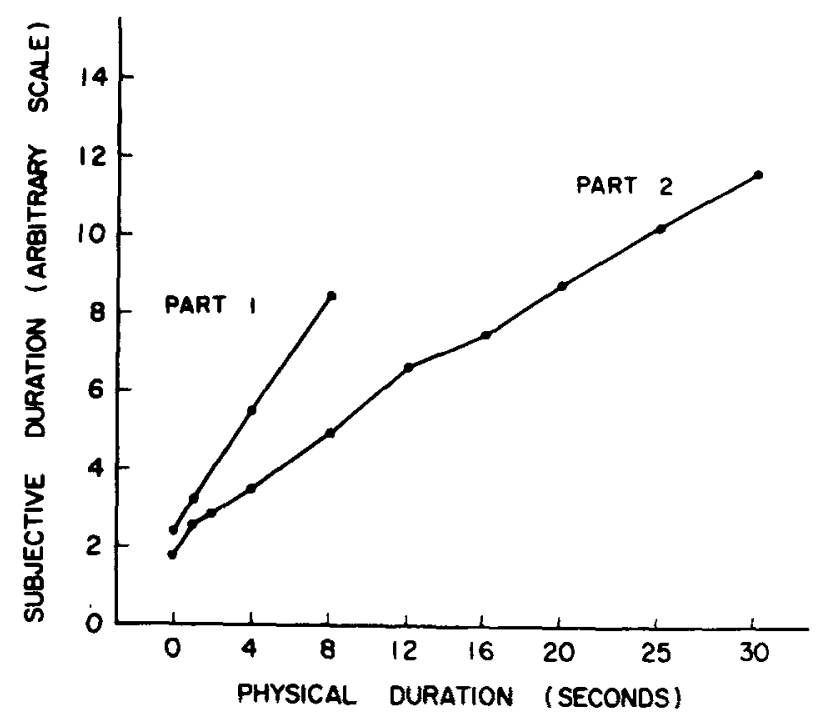

Figure 3. Psychophysical time function from Parts 1 and 2. Subjective duration is plotted against objective duration. 
is different because these values are estimated as marginal means of the factorial design, that is, as mean curves in Figures 1 and 2 . (It may be noted, incidentally, that a simple zero adjustment is available that would transform both interval scales into ratio scales by taking advantage of the fact, evident in Figures 1 and 2, that zero objective time corresponds to zero subjective time.) The unit is different because the total time range was different in the two parts. Interval scales may, of course, have arbitrary zero and unit. The important points are that both scales are interval scales by the validity criterion of the integration model, and that the two scales are consistent since each is a linear function of the other.

\section{DISCUSSION}

\section{The Psychophysical Function}

The present results indicate that the psychophysical function for perceived duration is very nearly linear over the range 0 to $30 \mathrm{sec}$. This conclusion, it should be recognized, rests on the use of the adding model to provide a validational base for stimulus scaling; although this model may seem prosaic in itself, it plays a vital theoretical role.

With two major exceptions that used the method of fractionation and are discussed below, previous work has also claimed to find a near-linear relation between perceived and actual duration (e.g., Clausen, 1950; Gregg, 1951; Ross \& Katchmar, 1951). The results of these studies are difficult to interpret, however, because each rests upon an unsupported assumption that the overt response was an interval scale of subjective duration. That assumption may or may not have been correct in the given experimental conditions, and in general it needs to be justified explicitly.

Two studies that have used the method of magnitude estimation have also claimed to find a linear or near-linear relation between perceived and actual duration (Stevens \& Galanter, 1957, Figure 2; Stevens \& Greenbaum, 1966). The interpretation of these results rests similarly on the assumption that the magnitude estimation response provides an interval (or ratio) scale of perceived duration. Stevens (e.g., 1974) has attempted to provide various criteria to support this assumption, but the validity of these attempts has been questioned on logical grounds by various writers (see Anderson, 1974a, Section IV.B.5) and on empirical grounds as well (Anderson, 1972, 1974b; Weiss, 1972, 1975).

The method of fractionation has been used in much of the work on the psychophysical function of duration. This method has certain peculiarities, however, that deserve separate comment. The main points of the following analysis also apply to the use of fractionation on other psychophysical dimensions.

\section{The Method of Fractionation}

In the method of fractionation, the subject adjusts a variable duration under instruction to produce some specified fraction of a standard duration. Denote the objective and subjective values of the variable duration by $\mathrm{V}$ and $\mathrm{v}$, respectively, those of the standard duration by $S$ and $s$, and let $f$ denote the subjective value of the fraction specified in the instructions. In the subjective metric, the model for the fractionation task is simply

$$
v=\text { fs. }
$$

The fractionation model is not readily analyzable because none of the three terms in this equation is observable. However, if it is assumed that the psychophysical function is a power function with exponent $n$, so that

$$
\mathrm{v}=\mathrm{V}^{\mathrm{n}}, \mathrm{s}=\mathrm{S}^{\mathrm{n}} \text {, }
$$

then substituting into Equation 2 and taking nth roots of both sides yields a linear relation among observable variables in the physical metric:

$$
\mathbf{V}=\mathrm{f}^{1 / \mathrm{n}} \mathrm{S} \text {. }
$$

Under this assumption, the observed variable duration should be proportional to the standard duration, regardless of the value of the exponent in the psychophysical function. This striking relation, due to Ekman (1958), has been well supported in virtually every study that has used the fractionation technique for judgments of duration. Thus, existing data support the joint assumption that the underlying fractionation model of Equation 2 is correct, and that the psychophysical function is a power function.

In this power function, however, the exponent is indeterminate. To determine the exponent using only fractionation data requires some further assumptions. Two approaches have been tried, based on arbitrary assumptions about the value of $n$, or about the value of $f$.

Early work on this problem (e.g., Gregg, 1951; Ross \& Katchmer, 1951) rested on the implicit assumption that the psychophysical function was linear, that is, that $\mathrm{V}$ was a linear function of $\mathrm{v}$ with $\mathbf{n}=1$. Since that assumption is false for other dimensions, such as heaviness (Anderson, 1972) and grayness (Weiss, 1972, 1975), it is arbitrary and requires explicit justification for duration. The present experiments illustrate how the linearity assumption can be justified using functional measurement.

Later work using the fractionation method (Björkman \& Holmkvist, 1960; Frankenhaeuser, 
1960) has taken explicit account of the underlying fractionation model. Their results show that fractionation of duration does follow the model of Equation 2, and that the psychophysical function is a power function. To determine the exponent of the power function, however, both articles make the arbitrary assumption that $f=1 / 2$, that is, that the instructed fractionation ratio was the subjectively effective ratio.

Both of these articles report markedly nonlinear psychophysical functions. Frankenhaeuser reports exponents of 1.87 in a normal condition, and 2.30 for subjects under radial acceleration. The exponents reported by Björkman and Holmkvist are smaller, but still substantially above 1. Their conclusions, therefore, disagree sharply with the present finding of a linear psychophysical function. The source of this disagreement presumably lies in their assumption that $f=1 / 2$; the present finding of a linear psychophysical function implies that $\mathrm{n}=1$, and that would imply $f>1 / 2$ in these studies.

A more extended discussion of fractionation is given in Anderson (1974, Section III.B.2). The general conclusion reached there is that fractionation has little value for scaling purposes, but should instead be studied for its intrinsic interest as a judgmental process.

\section{Applications}

Subjective duration does not have the same phenomenological immediacy that is found in other psychophysical situations, such as heaviness or grayness. As a consequence, the psychophysical function for duration is probably of less interest in itself than for its dependence upon the events that define the duration. It is known, for example, that a "filled" duration seems longer than an "empty" duration, and just this kind of problem has been at issue in much of the research on subjective duration (see, e.g., Ornstein, 1970; Thomas \& Brown, 1974).

Functional measurement may be useful in further study of the effects of event structure on perceived duration because it can provide a validated interval response measure. For example, the present total duration task could be used with the duration defined by an arbitrary sequence of any desired structure. Also, a subtracting model could be applied in a similar way to judgments of the difference between two durations. If these models are successful, then the data provide interval scales of subjective duration of the events as they operate within a given task. Interactions of time and distance could be treated in a similar way (Anderson, 1974, Section II.B.12).

A variant of the present experimental design is of particular interest. Suppose that subjects experience a series of events only once, and are later asked to judge the total duration of various pairs of the events. In this case, the response model would be the same as that in Equation 1, but the same stimuli could be used for both row and column factors. Using this extension of the present design, it would be possible to study a number of factors identified by Doob (1971) as being especially relevant to the study of subjective duration: the effects of internal cognitive states on impressions of duration, the perceived duration of very long events, the effect of the subject's awareness that he will be making a duration judgment before he experiences an event, the effects of event complexity, and finally, the effects of the passage of time on the judgment of duration.

\section{REFERENCES}

Anderson, N. H. Cross-task validation of functional measurement. Perception \& Psychophysics, 1972, 12. 389-395.

ANderson, N. H. Algebraic models in perception. In E. C. Carterette \& M. P. Friedman (Eds.), Handbook of perception (Vol. 2). New York: Academic Press, 1974. (a)

ANDERson. N. H. Cross-task validation of functional measurement using judgments of total magnitude. Joumal of Experimental Psychology, 1974, 102, 226-233. (b)

Buörkman, M., \& Holmkvist, 0 . The time-order error in the construction of a subjective time scale. Scandinavian Journal of Psychology, 1960, 1, 7-13.

Clausen. J. An evaluation of experimental methods of time judgments. Joumal of Experimental Psychology, 1950, 40, $756-762$.

Doos, L. S. Patterning of time. New Haven: Yale University Press, 1971.

Exman. G. Two generalized ratio scaling methods. Journal of Psychology, 1958, 45, 287-295.

Fraisse, P. The psychology of time. New York: Harcourt. Brace. 1963.

Frankenhaeuser, M. Subjective time as affected by gravitational stress. Scandinavian Jourmal of Psychology, 1960, 1, 1-6.

GREGG, L. W. Fractionation of temporal intervals. Journal of Experimental Psychology, 1951, 42, 307-312.

Ornstein, R. E. On the experience of time. Baltimore: Penguin Books, 1970.

Ross, S., \& KatchmaR, L. The construction of a magnitude function for short time-intervals. American Journal of Psycho$\log y, 1951.64,397.401$.

Stevers, S. S. Perceptual magnitude and its measurement. In E. C. Carterette \& M. P. Friedman (Eds.), Handbook of percep. tion (Vol. 2). New York: Academic Press, 1974.

Stevens. S. S. \& \& Galanter, E. H. Ratio scales and category scales for a dozen perceptual continua. Journal of Experimental Psychology, 1957, 54, 377-411.

Stevens, S. S., \& Greenbaum, H. Regression effect in psychophysical judgment. Perception \& Psychophysics, 1966, 1, 439-446.

Thomas, E. A. C., \& Brown, I. Time perception and the filledduration illusion. Perception \& Psychophysics, 1974, 16, 449-458.

WEISS, D. J. Averaging: An empirical validity criterion for magnitude estimation. Perception \& Psychophysics, 1972, 12, 385-388.

WEIss, D. J. Quantifying private events: A functional measurement analysis of equisection. Perception \& Psychophysics, 1975, 17, 351-357.

(Received for publication June 20, 1975; revision accepted June 15.1976. ) 